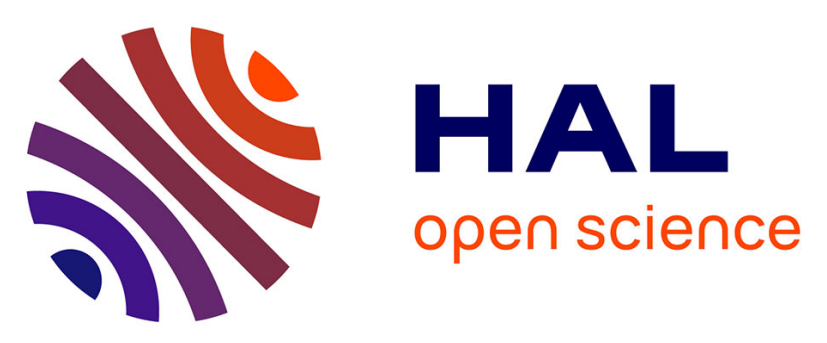

\title{
Numerical modeling of nominal and stray waves in birefringent interferometers: application to large-field-of-view imaging Fourier transform spectrometers
}

Hervé Sauer, Armande Pola Fossi, Yann Ferrec, Nicolas Guerineau, Jean

Minet, Jean Taboury, Pierre Chavel

\section{To cite this version:}

Hervé Sauer, Armande Pola Fossi, Yann Ferrec, Nicolas Guerineau, Jean Minet, et al.. Numerical modeling of nominal and stray waves in birefringent interferometers: application to large-fieldof-view imaging Fourier transform spectrometers. Applied optics, 2018, 57 (31), pp.9488-9498. 10.1364/AO.57.009488 . hal-01925659

\section{HAL Id: hal-01925659}

https://hal-iogs.archives-ouvertes.fr/hal-01925659

Submitted on 29 Nov 2018

HAL is a multi-disciplinary open access archive for the deposit and dissemination of scientific research documents, whether they are published or not. The documents may come from teaching and research institutions in France or abroad, or from public or private research centers.
L'archive ouverte pluridisciplinaire HAL, est destinée au dépôt et à la diffusion de documents scientifiques de niveau recherche, publiés ou non, émanant des établissements d'enseignement et de recherche français ou étrangers, des laboratoires publics ou privés. 


\title{
Numerical modeling of nominal and stray waves in birefringent interferometers: Application to large field of view imaging Fourier transform spectrometers
}

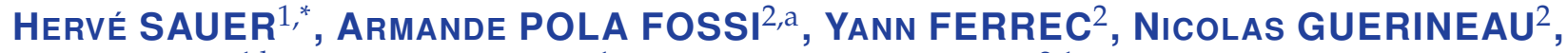 \\ Jean MINET ${ }^{1, b}$, Jean TABOURY ${ }^{1, c}$, And Pierre ChaVEL ${ }^{3,1}$ \\ ${ }^{1}$ Laboratoire Charles Fabry, Institut d'Optique Graduate School, CNRS, Université Paris-Saclay, 2 avenue Augustin Fresnel, 91127 Palaiseau, France \\ ${ }^{2}$ ONERA, chemin de la Hunière, 91123 Palaiseau, France \\ ${ }^{3}$ Laboratoire Hubert Curien, Université de Lyon, Université Jean Monnet de Saint Etienne, CNRS UMR 5516, 42000 Saint Etienne, France \\ *Corresponding authors: herve.sauer@institutoptique.fr
}

Manuscript AO 340366 - Revised version - Compiled October 9, 2018

\begin{abstract}
Birefringent interferometers are often used for compact static Fourier transform spectrometers. In such devices, several uniaxial birefringent parallel or prismatic plates are stacked, with their optical axes set so that there is an efficient coupling from ordinary to extraordinary and extraordinary to ordinary eigenmodes of two successive plates. Such coupling, aside from few particular cases, is however not perfect, an effect that may adversely affect performance. In order to help the design and the tolerancing of these interferometers, we have developed a numerical modeling, based on the propagation of plane waves inside and through the interface of birefringent media. This tool evaluates the traveled optical path length and the amplitude of the different polarization modes, enabling to predict both the optical path differences on the interferometer outputs and the unwanted coupling strengths and related stray wave amplitudes. The tool behavior is illustrated on Savart and Double-Wollaston interferometers, and compared with experimental characterization of a calcite Double-Wollaston prism. @ 2018 Optical Society of America
\end{abstract}

OCIS codes: (260.1440) Birefringence; (120.6200) Spectrometers and spectroscopic instrumentation; (110.4234) Multispectral and hyperspectral imaging; (120.0280) Remote sensing and sensors; (120.4570) Optical design of instruments; (300.6300) Spectroscopy, Fourier transforms; (220.4830) Systems design.

http://dx.doi.org/10.1364/ao.XX.XXXXXX

\section{INTRODUCTION}

Imaging Static Fourier Transform Spectrometry (SFTS) is a good approach for airborne or spaceborne hyperspectral imaging [16], especially thanks to its sturdiness (no moving part except the carrier or the scan turret) and its high flux collection efficiency [7]. The formerly cited references give detailed explanations on the setup and underlying optical principles of such instruments; the key point is to superimpose quasi-linearly shaped interference fringes on the scene image. Efficient large étendue imaging instruments usually rely on a shearing interferometer, most often a cyclic interferometer [1-4] or a roof-prism mirrors Michelson interferometer [5, 6, 8]. For more compact setups, birefringent shearing interferometers like Savart plates [9-11]

\footnotetext{
a Armande Pola Fossi is now with Thales LAS France SAS, 2 avenue GayLussac, 78990 Élancourt, France.

b Jean Minet is now with Koheron, Centre Scientifique d'Orsay, Bât.503, 91400 Orsay, France.

c Jean Taboury is now retired.
}

or Double-Wollaston prism devices $[12,13]$ are often used, either for imaging spectrometry or for non-imaging spectrometry. Other designs with birefringent interferometers, aside from lateral shearing interferometers, have also been developed for Fourier transform spectrometry [14-20] or polarimetry [21, 22]. Most of these birefringent interferometers are made of uniaxial materials that physically split an incident beam into two orthogonally polarized beams (ordinary and extraordinary ones), bringing different optical lengths when propagating through the system. The optical path difference (OPD) of two such beams, which interfere on a given sensor point, changes with the localization of that point, leading to the interference fringe pattern on the scene image. However, with only one monolithic birefringent plate or prism, one optical path is always significantly longer than the other one, and the zero OPD cannot be reached, which is harmful for FTS measurements. To overcome such a limitation, two or more birefringent plates or prisms are used with suitable birefringent material properties and orientations 
in order to allowthe zero OPD to be reached for at least one direction of the incident wave inside the field of view. In most cases, the same material is used for all birefringent elements, and compensation is obtained by exchanging the ordinary and extraordinary nature of each beam when it enters the next birefringent material. Such an oe or eo exchange, however, is perfect only for specific propagation and polarization directions of the beam and specific birefringent material orientations. In most practical cases (skew incident rays, errors in manufacturing or assembly of birefringent plates or prisms, and so on), beam coupling at the interface between adjacent birefringent materials is not perfect and stray waves may exist due to non-zero coupling between ordinary beam to ordinary beam (oo coupling) or extraordinary beam to extraordinary beam (ee coupling). ${ }^{1}$ Such stray waves or stray beams may alter the interferometer nominal behavior. In this paper, we investigate the amplitude and effect of such stray waves in order to be able to derive rules regarding usable field of view and required manufacturing and assembling tolerances for specific devices and applications. The following section will present the outline of the electromagnetic theory that allows us to define and compute transmission and reflection coefficients at the interface between two anisotropic or isotropic dielectric materials (including biaxial, uniaxial and also isotropic ones in any combination). These coefficients will then be used in section 3 to build an accurate model of the behavior of real birefringent devices including stray coupling between extraordinary and ordinary waves. Section 4 will use this model to predict the effect of stray waves on Savart plates or similar layered devices in one hand, and for the Double-Wollaston prism device in the second hand. Section 5 will present experimental observations of the stray waves effect on a Double-Wollaston interferometer.

\section{BACKGROUND ELECTROMAGNETIC MODAL AND $4 \times 4$ MATRIX FORMALISMS}

\section{A. Plane waves in birefringent media}

From an electromagnetic point of view, a (non-magnetic, nonoptically active, non absorbing) birefringent medium is characterized at a given vacuum wavelength $\lambda_{0}$ by the $3 \times 3$ real symmetric relative dielectric tensor that is diagonal in the frame $\left(X^{\prime}, Y^{\prime}, Z^{\prime}\right)$ of the birefringent material principal dielectric axes [23]:

$$
\left[\varepsilon_{r P}\right]=\left[\begin{array}{ccc}
\varepsilon_{x^{\prime}} & 0 & 0 \\
0 & \varepsilon_{y^{\prime}} & 0 \\
0 & 0 & \varepsilon_{z^{\prime}}
\end{array}\right]
$$

When all the principal dielectric constants $\varepsilon_{x^{\prime}, y^{\prime}, z^{\prime}}$ are identical, the medium is said to be isotropic, when they are all different, it is said to be biaxial and otherwise the medium is said to be uniaxial. For a given $(X, Y, Z)$ frame related to the device, this tensor is expressed as:

$$
\left[\varepsilon_{r}\right]=\mathbf{R}\left[\varepsilon_{r P}\right] \mathbf{R}^{-1}=\left[\begin{array}{ccc}
\varepsilon_{x x} & \varepsilon_{x y} & \varepsilon_{x z} \\
\varepsilon_{y x} & \varepsilon_{y y} & \varepsilon_{y z} \\
\varepsilon_{z x} & \varepsilon_{z y} & \varepsilon_{z z}
\end{array}\right]
$$

where $\mathbf{R}$ is the rotation matrix from the given frame to the principal axes frame of the birefringent material.

\footnotetext{
${ }^{1}$ For devices that use different birefringent materials with opposite birefringence signs, the nominal couplings are thus $e e$ and $o o$ and the stray couplings are $e o$ and oe.
}

According to [24-26], electromagnetic plane-wave propagation in an homogeneous anisotropic dielectric medium, basically described by Maxwell's equations, can be reformulated in a general way as a modal problem that involves $4 \times 4$ complex matrices acting on 4 components of the complex electromagnetic fields. This $4 \times 4$ matrix formalism can be further extended to describe coupling (i.e. generalized Fresnel transmission and reflection coefficients) between waves at the interface between two (isotropic or anisotropic) materials, and in a stack of anisotropic or isotropic parallel plates [25-29]. This sub-section summarizes our own implementation of that formalism, which we will use later on as a tool to study birefringent interferometers.

Inside a homogeneous (anisotropic or isotropic) medium, we look for waves satisfying the following conditions:

- They are monochromatic, of angular frequency $\omega=$ $2 \pi . c / \lambda_{0}$, where $\lambda_{0}$ is the wavelength in vacuum and $c$ the vacuum light velocity. We will assume an implicit $e^{-i \omega t}$ time dependance of the complex time varying fields. We note $k_{0}=2 \pi / \lambda_{0}$

- They can propagate in the medium, that is, they satisfy Maxwell's equations.

- The complex amplitude of their electric and magnetic fields can be written in the form of Eq.3, where $k_{x}$ and $k_{y}$ are arbitrarily fixed, and can be seen as the projection of the wavevector in the XY plane.

$$
\left\{\begin{array}{l}
\vec{E}(x, y, z)=\vec{E}(z) e^{i\left(k_{x} x+k_{y} y\right)} \\
\vec{H}(x, y, z)=\vec{H}(z) e^{i\left(k_{x} x+k_{y} y\right)}
\end{array}\right.
$$

In the following, we will note $k_{x}=n_{x} k_{0}$ and $k_{y}=n_{y} k_{0}$. Be careful that what we call $n_{x}$ and $n_{y}$ are not the principal refractive indices of the material $\sqrt{\varepsilon_{x^{\prime}}}$ and $\sqrt{\varepsilon_{y^{\prime}}}$.

With the assumptions of Eq. 3, Maxwell's equations $\vec{\nabla} \times \vec{E}=$ $i \omega \mu_{0} \vec{H}$ and $\vec{\nabla} \times \vec{H}=-i \omega \varepsilon_{0}\left[\varepsilon_{r}\right] \vec{E}$ lead to (with $Z_{0}=\mu_{0} c=$ $\sqrt{\mu_{0} / \varepsilon_{0}}$ the vacuum impedance) :

$$
\frac{\partial \vec{\psi}}{\partial z}=i k_{0} \mathcal{M} \vec{\Xi}
$$

where $\vec{\psi}(z)=\left[\begin{array}{l}E_{x}(z) \\ E_{y}(z) \\ Z_{o} H_{x}(z) \\ Z_{0} H_{y}(z)\end{array}\right]$ and $\vec{\Xi}(z)=\left[\begin{array}{l}E_{x}(z) \\ E_{y}(z) \\ E_{z}(z) \\ Z_{0} H_{x}(z) \\ Z_{0} H_{y}(z) \\ Z_{0} H_{z}(z)\end{array}\right]$ and $\mathcal{M}$ is a $4 \times 6$ matrix whose elements are the following ${ }^{2}$ : $\left[\begin{array}{cccccc}0 & 0 & n_{x} & 0 & 1 & 0 \\ 0 & 0 & n_{y} & -1 & 0 & 0 \\ -\varepsilon_{y x} & -\varepsilon_{y y} & -\varepsilon_{y z} & 0 & 0 & n_{x} \\ \varepsilon_{x x} & \varepsilon_{x y} & \varepsilon_{x z} & 0 & 0 & n_{y}\end{array}\right]$.

\footnotetext{
${ }^{2}$ We used $Z_{0} H_{x \mid y}$ instead of $H_{x \mid y}$ in the $\vec{\psi}$ vector in order to get simpler and better conditionned matrices in the following.
} 
The two same Maxwell's equations also allow to express $H_{z}$ from $E_{x}$ and $E_{y}$ and $E_{z}$ from $H_{x}, H_{y}, E_{x}$ and $E_{y}$. We can thus write that $\vec{\Xi}(z)=\mathcal{A} \vec{\psi}(z)$ with $\mathcal{A}$ being the following $6 \times 4$ matrix:

$$
\mathcal{A}=\left[\begin{array}{cccc}
1 & 0 & 0 & 0 \\
0 & 1 & 0 & 0 \\
-\frac{\varepsilon_{z x}}{\varepsilon_{z z}} & -\frac{\varepsilon_{z y}}{\varepsilon_{z z}} & \frac{n_{y}}{\varepsilon_{z z}} & -\frac{n_{x}}{\varepsilon_{z z}} \\
0 & 0 & 1 & 0 \\
0 & 0 & 0 & 1 \\
-n_{y} & n_{x} & 0 & 0
\end{array}\right]
$$

Therefore, the waves that follow Eq. 3 and the Maxwell's equations in the medium, follow a first-order linear ordinary differential equation [26]:

$$
\frac{\partial \vec{\psi}}{\partial z}=i k_{0} \mathbf{M} \vec{\psi} \quad \text { with } \quad \mathbf{M}=\mathcal{M} \mathcal{A} \quad \text { a } 4 \times 4 \text { matrix }
$$

The general solution of such an homogeneous constant coefficient linear ordinary differential equation is a linear combination of elementary solutions, usually called "modes" in the electromagnetic propagation context, that can be expressed as:

$$
\vec{\psi}(z)=\sum_{\ell=1}^{4} \gamma_{\ell} e^{i k_{0} n_{z, \ell} z} \vec{\psi}_{p, \ell}
$$

with $\vec{\psi}_{p, \ell}$ the four eigenvectors of matrix $\mathbf{M}$, and $n_{z, \ell}$ its four eigenvalues. $\gamma_{\ell}$ are free complex coefficients. Combined with Eq. 3, this shows that the solutions are a superposition of four plane waves. The $k_{z, \ell} \triangleq n_{z, \ell} \times k_{0}$ quantities represent the $\mathrm{Z}$ component of the wavevectors of the plane waves (or "modes") that propagate in the medium and the associated eigenvectors represent the $X Y$ components (in the used frame) of the $\vec{E}$ and $\vec{H}$ of the modes. Eq. 7 can be written in a matrix form by introducing $\mathbf{V}$, a matrix whose columns are the eigenvectors $\vec{\psi}_{p, \ell}$, and a diagonal matrix $\mathbf{D}(z)$ linked to the eigenvalues:

$$
\vec{\psi}(z)=\mathbf{V} \mathbf{D}(z) \vec{\gamma}
$$

with $\mathbf{V}=\left[\vec{\psi}_{p, 1}\left|\vec{\psi}_{p, 2}\right| \vec{\psi}_{p, 3} \mid \vec{\psi}_{p, 4}, \mathbf{D}(z)=\right.$
$\left[\begin{array}{cccc}e^{i k_{z, 1} z} & 0 & 0 & 0 \\ 0 & e^{i k_{z, 2} z} & 0 & 0 \\ 0 & 0 & e^{i k_{z, 3} z} & 0 \\ 0 & 0 & 0 & e^{i k_{z, 4} z}\end{array}\right]$ and $\vec{\gamma}=\left[\begin{array}{c}\gamma_{1} \\ \gamma_{2} \\ \gamma_{3} \\ \gamma_{4}\end{array}\right]$.

Coefficients $\gamma_{\ell}$ are the amplitudes of the eigenmodes. Note that $\mathbf{V}$ and $k_{z, \ell}$ depend on $\vec{k}_{/ /}=\left[n_{x} \cdot k_{0}, n_{y} \cdot k_{0}, 0\right]$.

\section{B. Mode normalization and sorting}

From a practical point of view, eigenvalues and eigenvectors can be numerically computed with standard linear algebra numerical computing tool (we used "eig" in Matlab ${ }^{\circledR}$ ). The order and the normalization of the computed eigenvectors are however arbitrary relatively to physical meanings. For the case of nonevanescent waves in non absorbing media, we use the real part $\Re\left(S_{z}\right)$ of the $z$ component of the complex Poynting vector $S_{z}$
[30] to normalize the $\vec{\psi}_{p, \ell}$ vectors. Normalization is done by applying a complex factor on each eigenvector so that $\left|\Re\left(S_{z}\right)\right|=1$ and $E_{x}$ be real positive (or $E_{y}$ be real positive if $E_{x}$ is zero). This implies that all modes represent plane waves that flow $\pm 1 \mathrm{~W} / \mathrm{m}^{2}$ through surfaces normal to the $Z$ axis.

About the sorting of the mode, there are always two modes flowing towards ascending $z\left(\Re\left(S_{z}\right)>0\right)$ and two modes that flow toward decreasing $z\left(\Re\left(S_{z}\right)<0\right)$, allowing identification of left-to-right and right-to-left traveling modes. In the case of a uniaxial material, we can further identify the modes by computing $n_{z, \ell}^{2}+n_{x}^{2}+n_{y}^{2}$ and comparing it to $n_{o}^{2}$ where $n_{o}$ is the ordinary refractive index of the material; such comparison matches within the double-precision numerical computing error for two modes (ordinary mode that travels at ascending $z$ " $o+"$ and decreasing $z$ " $o-")$ and disagrees for the two others (extraordinary mode that travels at ascending $z$ " $e+"$ and decreasing $z$ "e-"). In case of degeneracy in the eigenvalues, which arises for an isotropic material or some configurations for an anisotropic material and introduces new arbitrariness on the eigenvectors, we enforce transverse electric (TE) and transverse magnetic (TM) polarization for the modes.

As we never use biaxial materials, but only uniaxial or isotropic ones, we will now restrict our description to this situation. The $\vec{\psi}_{p, \ell}$ column vectors that built the $V$ matrix are then sorted in the $(e+, o+, e-, o-)$ order for non-degenerate uniaxial cases, and in the $(T E+, T M+, T E-, T M-)$ order for the isotropic or degenerate uniaxial cases. For the sake of simplicity in the notations, we will always name the modes by $e+, o+, e-$ and $o-$, being understood that " $e$ " (respectively " $o$ ") means "extraordinary" (resp. "ordinary") for non degenerate uniaxial case or TE (resp. TM) for degenerate or isotropic cases.

It is worth noting that these modes fulfill an interesting property regarding the complex Poynting vector $\vec{S}=\frac{1}{2} \vec{E} \times \overrightarrow{H^{*}}$ (where $\times$ stands for cross-product and ${ }^{*}$ for complex conjugate) [30]. In the general case, the Poynting vector for the superposition of two electromagnetic fields $\left(\overrightarrow{E_{A}}, \overrightarrow{H_{A}}\right)$ and $\left(\overrightarrow{E_{B}}, \overrightarrow{H_{B}}\right)$, $\vec{S}_{A+B}=\frac{1}{2}\left(\overrightarrow{E_{A}}+\overrightarrow{E_{B}}\right) \times\left(\overrightarrow{H_{A}}+\overrightarrow{H_{B}}\right)^{*}$ is not equal to the sum of the individual Poynting vectors $\overrightarrow{S_{A}}=\frac{1}{2} \overrightarrow{E_{A}} \times{\overrightarrow{H_{A}}}^{*}$ and $\overrightarrow{S_{B}}=\frac{1}{2} \overrightarrow{E_{B}} \times \overrightarrow{H_{B}}$. However, this holds for the real part of the complex Poynting vector $z$ components for the modes fields (for non evanescent modes in non absorbing media), where

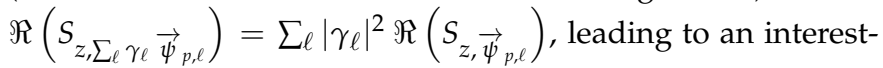
ing property of power flux decoupling at $X Y$ interfaces for the four modes.

\section{Coupling of the waves at the interface between two birefrin- gent media}

We now consider the interface between two semi-infinite birefringent media A and B. The current frame of reference is defined so that this interface is the plane $z=0$, and we have an incident wave with a known $\vec{k} / /$. The continuity of the tangential components of $\vec{E}$ along with Eq. 3 imply that $\vec{k}_{/ /}$is the same in medium B as in medium A. According to the definition of $\vec{\psi}$, and thanks to the continuity of the tangential component of the electric and magnetic vectors at the interface [31], we have:

$$
\vec{\psi}_{A}=\vec{\psi}_{B}
$$


and thus

$$
\mathbf{V}_{\mathbf{A}}\left[\begin{array}{c}
\gamma_{e+, A} \\
\gamma_{o+, A} \\
\gamma_{e-, A} \\
\gamma_{o-, A}
\end{array}\right]=\mathbf{V}_{\mathbf{B}}\left[\begin{array}{c}
\gamma_{e+, B} \\
\gamma_{o+, B} \\
\gamma_{e-, B} \\
\gamma_{o-, B}
\end{array}\right]
$$

Since matrix $\mathbf{V}$ is defined by the dielectric tensor and by $\vec{k} / /$, we thus know $\mathbf{V}_{\mathbf{A}}$ and $\mathbf{V}_{\mathbf{B}}$. It is therefore possible to find the amplitudes of the electromagnetic modes in medium B knowing the ones in medium A. Thanks to the normalization of the modes (see previous subsection), we can calculate the transmission and reflection coefficients for this interface between media A and B, by solving the following equation:

$$
\left[\begin{array}{c}
1 \\
0 \\
r_{e, e} \\
r_{e, o}
\end{array}\right]=\mathbf{V}_{\mathbf{A}}^{-1} \mathbf{V}_{\mathbf{B}}\left[\begin{array}{c}
t_{e, e} \\
t_{e, o} \\
0 \\
0
\end{array}\right] \text { and }\left[\begin{array}{c}
0 \\
1 \\
r_{o, e} \\
r_{o, o}
\end{array}\right]=\mathbf{V}_{\mathbf{A}}^{-1} \mathbf{V}_{\mathbf{B}}\left[\begin{array}{c}
t_{o, e} \\
t_{o, o} \\
0 \\
0
\end{array}\right]
$$

where $t_{e, e}$ and $t_{e, o}$ are the generalized Fresnel complex transmission coefficients from $e$-mode to modes $e$ and $o$, and $t_{o, e}$ and $t_{o, o}$ are the generalized Fresnel complex transmission coefficients from $o$-mode to modes $e$ and $o$, and the same for the generalized Fresnel complex reflection coefficients $r_{e, e}, r_{e, o}, r_{o, e}$ and $r_{o, o}$. The two equations above simply state that we enforce an amplitude of 1 for respectively the $e+$ or $o+$ modes in material A, and we get in both cases the reflected waves in $e-$ and $o-$ modes in material A and the transmitted waves in the $e+$ and $o+$ modes in material B. Each equation in Eq. 11 can be rewritten as a standard set of linear equations with four unknowns easy to solve. This gives an effective and efficient way to compute these generalized Fresnel complex coefficients between two semi-infinite anisotropic or isotropic materials.

Thanks to the power flux decoupling of modes described at the end of the previous sub-section, we can also meaningfully define generalized energetic Fresnel coefficients $T_{e, e}, T_{e, o}, T_{o, e}$, $T_{o, o}, R_{e, e}, R_{e, o}, R_{o, e}$ and $R_{o, 0}$, by the ratio of the real-part of the $z$-component of the Poynting vector for the considered transmitted or reflected mode over the real-part of the $z$-component of the Poynting vector for the considered incident mode. Due to the Poynting normalization of modes used, these energetic coefficients are simply the squared modulus of the corresponding former complex transmission and reflection coefficients.

\section{MODELING OF THE REAL BEHAVIOR OF BIREFRIN- GENT IMAGING INTERFEROMETERS}

Our goal is to model the propagation of the beam or of the waves inside a birefringent imaging interferometer in order to predict interference fringe shape and possible deviation from the nominally expected behavior. For device built from a stack of birefringent plates with all interfaces parallel to each other, Eq. 8 and Eq. 10 allow to connect amplitudes of the modes on the left side to the amplitudes of the modes on the right size of the whole device with a matrix $\mathbf{P}$ defined by a matrix product like $\mathbf{V}_{\mathrm{A}}^{-1} \mathbf{V}_{\mathrm{B}} \mathbf{D}_{\mathrm{B}}\left(e_{\mathrm{B}}\right)^{-1} \mathbf{V}_{\mathrm{B}}^{-1} \mathbf{V}_{\mathrm{C}} \mathbf{D}_{\mathrm{C}}\left(e_{\mathrm{C}}\right)^{-1} \ldots$ for materials A, B, C, .. of thicknesses $e_{\mathrm{B}}, e_{\mathrm{C}}, \ldots$ This is the so-called $4 \times 4$ matrix method [25-29]. It is able to fully predict the output for a given input wave, taking simultaneously into account all internal (nominal and stray) transmissions and reflections at all interfaces, including multiple reflections between interfaces.

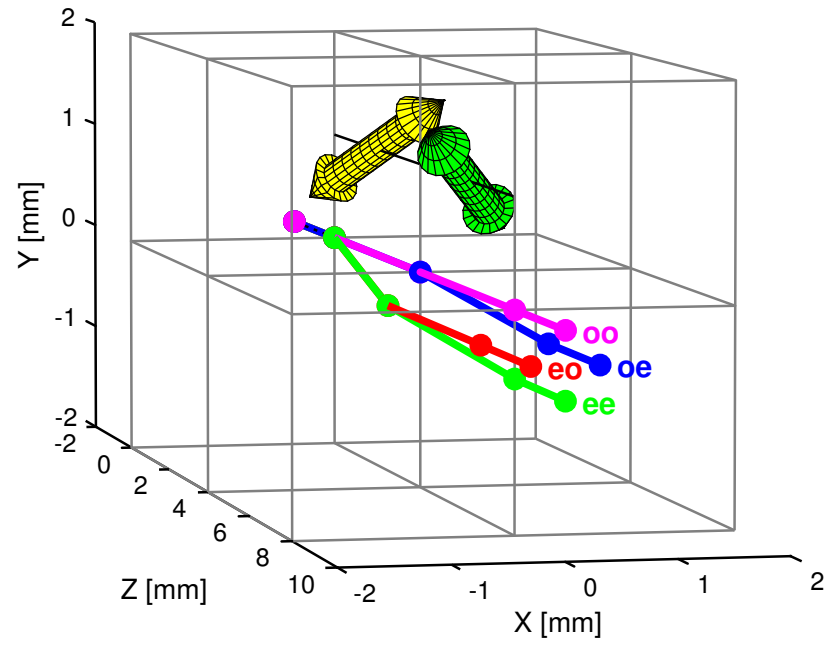

Fig. 1. Savart plates, with the nominal (oe in blue and $e o$ in red) and stray (oo in magenta and ee in green) rays. 3D arrows show the orientation of the optical axis of the anisotropic uniaxial materials (calcite here). Note that the drawn rays follow the Poynting vector direction, which is not usually identical to the wavevector direction. Such a configuration with the nominal rays that exit at the same $Y$ coordinates (for normal incidence) and different $X$ coordinates would give mainly vertical (along Y) nominal fringes. For normal incidence, the stray rays exit at the same $X$ coordinates and have the same spacing along $\mathrm{Y}$ than the spacing of nominal rays along $\mathrm{X}$.

However, such a global approach does not give good insight on the physical behavior of the device, as the computed output contains the effect of nominal and stray waves as a whole that cannot be unmixed. Moreover this method cannot be extended to devices that use prisms, such as the Double-Wollaston or the Savart plate device with manufacturing defects that break the parallelism of its interfaces. Therefore, in order to get a better insight of the birefringent interferometer behavior and to be able to tackle arbitrary stacks of parallel and/or wedged birefringent plates, a case which to our knowledge has seldom been discussed in the literature, or only partly [12, 32, 33], we prefer to use a slightly approximated ("geometrical") method that deals with rays or beams that travel in the device following the Poynting vector direction of a given mode in each material. (We, thus, neglect multiple reflections inside the device. However, as the index difference is low at birefringent/birefringent interfaces (usually less than $\left|n_{e}-n_{o}\right|$ ), and external interfaces with air are usually anti-reflection coated, reflected waves are weak, and multiple reflection effects are usually negligible in practice).

For such a "geometrical" modeling, we characterize each beam or ray by an ordinary $(o)$ or extraordinary $(e)$ attribute in each anisotropic uniaxial material it propagates across (it doesn't matter in isotropic material as modes are degenerated). For instance, in a two-plate interferometer like the Savart device, 4 beams can be defined if we do not take into account reflections: $e e, e o$, oe and $o o$. At each internal interface, we compute the generalized complex and energetic transmission Fresnel coefficients as explained at the end of the previous section, and we can thus compute the global complex and energetic coefficients of transmission of each beam, excluding Fresnel losses at the 
device entrance and exit interfaces with the isotropic air ${ }^{3}$. As we also know the wavevector of each mode, we can besides compute the optical path length traveled by each beam, and therefore predict the interference state between the exiting plane waves for a given (coherent) input beam.

That method has been implemented with a set of Matlab ${ }^{\circledR}$ functions grouped in a toolbox we called "SELBir"4. A (quite detailed) overview of our computation algorithm for the simulation of stacks of parallel or wedged birefringent plates is given in a synoptic flowchart on Fig. 2.

Fig. 1 shows the example of the Savart interferometer, made of two plates in calcite, each being of thickness $e=4 \mathrm{~mm}$, with optical axis slanted at $\theta=45^{\circ}$ from $Z$ axis for both plates, and optical axis projection in XY plane tilted at $\psi_{1}=45^{\circ}$ and $\psi_{2}=135^{\circ}$ from the $X$ axis for respectively the first and second plates. Fig. 3 gives the nominal optical path difference (that is, between oe and $e o$ beams) at $\lambda_{0}=500 \mathrm{~nm}$ for different incident directions $\left(\alpha_{x}, \alpha_{y}\right)$ on $\mathrm{a} \pm 30^{\circ} \times \pm 20^{\circ}$ field of view. The OPD varies mainly with $\alpha_{x}$ as could be anticipated from the direction of the lateral shearing between the oe and eo rays (see Fig. 1). For interferometers like the Savart plate made of a stack of perfect parallel plates surrounded with an isotropic medium, the optical path difference can be calculated using the optical path difference of a plate given by Françon and Mallick or Veiras et al. [34, 35] . In the case of a Savart interferometer with two plates of equal thickness and optical axes oriented as in the example above, the analytical expression of the OPD at the second order commonly used is:

$$
\begin{aligned}
\frac{O P D\left(\alpha_{x}, \alpha_{y}\right)}{e}= & \frac{a^{2}-b^{2}}{a^{2}+b^{2}} \frac{\sqrt{2} \cdot \tan \alpha_{x}}{\sqrt{1+\tan ^{2} \alpha_{x}+\tan ^{2} \alpha_{y}}} \\
& -\frac{\sqrt{2} \cdot a^{2} \cdot\left(a^{2}-b^{2}\right)}{\left(a^{2}+b^{2}\right)^{3 / 2}} \frac{\tan \alpha_{x} \cdot \tan \alpha_{y}}{1+\tan ^{2} \alpha_{x}+\tan ^{2} \alpha_{y}}
\end{aligned}
$$

with $e$ the thickness of each plate, $\alpha_{x}$ and $\alpha_{y}$ the field angles in the air (precisely defined by $\tan \alpha_{x}=k_{x} / k_{z}$ and $\tan \alpha_{y}=k_{y} / k_{z}$ ), $a=1 / n_{e}, b=1 / n_{o}\left(n_{e}\right.$ and $n_{o}$ being the principal extraordinary and the ordinary refractive indices). Françon and Mallick or Veiras et al. also give an exact closed-form expression. We have therefore compared this latter with the OPD calculated on Fig. 3. The two computations match as expected to a relative error less than $10^{-12}$ for double-precision floating point computations.

On Fig. 4, we have plotted the evolution with the field of view of the energetic transmission coefficient at the interface between the two birefringent plates of the Savart interferometer, in two cases: On Fig. 4.a, we have plotted $T_{o e}$, which is related to one of the two nominal waves in the interferometer; on Fig. 4.b, we have plotted $T_{o o}$, which is related to one of the two stray waves. Such transmission coefficients represent the transmission of the interferometer, excluding Fresnel losses at the entrance and exit interfaces with air of the whole device, as explained in the footnote ${ }^{3}$. The first one, $T_{o e}$, characterizes the strength of

\footnotetext{
${ }^{3}$ As already stated, device external interfaces with air are usually anti-reflection coated, leading to extremely low Fresnel losses when light enters or exits the device. Moreover Fresnel transmission coefficients at the first isotropic/birefringent interface depend on the incident beam polarization, and Fresnel transmission coefficients at the last birefringent/isotropic interface depend on the polarization of the exit mode considered in the last isotropic semi-infinite medium. Therefore, default computations consider them to be unity. All the analyses presented later in this paper have been performed in this way. This allows focusing on the main properties of birefringent interferometer due to the anisotropic/anisotropic interfaces. However our method can take into account, if required, Fresnel losses at entrance and exit interfaces (with no anti-reflection coating) for a given TE or TM polarization for the entrance beam and a given TE or TM analyze state on the exit beam.

${ }^{4}$ Interested readers can contact the corresponding author. (At present, the code is only documented in French).
}

the expected coupling at the birefringent material interface of the incident $o$-wave to a transmitted $e$-wave. The second coefficient, $T_{o o}$, characterizes the strength of the unwanted coupling of the incident $o$-wave to a transmitted $o$-wave. We can see on the graphics that, as expected, $T_{o e}$ is high and $T_{o o}$ is low. However, $T_{o e}$ is really close to 1 only in the center of the field, and may drop to value lower than 0.88 for some points at the edge of the $+20^{\circ} y$-field of view. In the same way, $T_{o o}$ is close to 0 only in the same central area of the field of view and increases as the field angles grow, reaching values greater than 0.12 at some points at the edge of the field of view. This shows that stray waves may carry more than $10 \%$ of the whole wave power in the interferometer. Results and conclusion are similar for $T_{e o}$ and $T_{e e}$ transmission coefficients. The properties of these stray waves are more thoroughly discussed in the next sections.

\section{THEORETICAL EFFECT OF STRAY WAVES ON THE INTERFEROGRAM}

\section{A. Case of Savart plate and other stacked parallel plate de- vices}

The Savart plate, as described in the previous section, is commonly used in spectropolarimetry and in astronomy as polarization analyzer [36-38], and it is thus a good example for our work. To be used as an imaging interferometer, this Savart plate is placed between polarizer and analyzer, both oriented within $\pm 45^{\circ}$ of the $X Y$ projection of the uniaxial material axes of the device (that is along the $X$ or the $Y$ direction for Fig. 1 geometry). In the general case, as schematically depicted by geometrical rays in Fig. 1, the linearly polarized incident plane wave that enters the Savart plate interferometer gives rise to four transmitted plane waves, $e o, o e$, oo and $e e$, which exit the Savart plate interferometer with the same direction and thus the same wavevector $\vec{k}_{i}$ than the incident wave, due to the conservation of $\vec{k}_{/ /}$at dielectric interfaces. These four plane waves superimpose coherently and form a unique resultant plane wave with wavevector $\vec{k}_{i}$. The resulting complex amplitude of the linearly polarized wave that leaves the analyzer is thus the sum of the complex amplitudes of four waves, $A=A_{e o}+A_{o e}+A_{e e}+A_{o o}$. In order to qualitatively understand how the intensity $I=|A|^{2}$ of that wave evolves with the incident direction, we can note that the linearly polarized incident plane wave splits in nearly equal intensity into the $e+$ and $o+$ modes of the first uniaxial material. Therefore, the modulus of the constituting wave complex amplitudes $A_{e o}, A_{o e}, A_{e e}$ and $A_{o o}$ are directly related to $\sqrt{T_{e o}}, \sqrt{T_{o e}}, \sqrt{T_{e e}}$, $\sqrt{T_{o o}}$ and their phases are given by the optical paths traveled by each wave inside the device. Interference between all these four waves will determine the intensity of the resultant exiting wave in that direction for a given incident direction, and, then, the appearance of the fringe field for a given field of view. This fringe field $I=A A^{*}$ involves thus six crossed terms $2 \Re\left(A_{\Xi \Theta} A_{\Xi^{\prime} \Theta^{\prime}}^{*}\right)$, where $\Xi, \Xi^{\prime}, \Theta$ and $\Theta^{\prime}$ stand for $e$ and $o$ and are able to represent the six pairs $(e o, o e),(e o, e e),(e o, o o),(o e, e e),(o e, o o)$ and $(e e, o o)$, and relies on the OPD evolution over the field of these pairs of waves.

The OPD map for the nominal interference between $e o$ and oe waves has already been presented on Fig. 3. On Fig. 5, we show the OPD map for interference between oe and oo waves. The gradient of the OPD is now no more along the $\mathrm{X}$ direction, but, as expected, along the $\left(\sim-45^{\circ}\right)$ direction that connects the oe and oo ray exit points in Fig. 1. We can moreover note that the zero OPD value is never reached, due to the fact that path difference 


\section{INPUT DATA SUMMARY :}

\begin{tabular}{|c|c|c|c|c|c|c|c|}
\hline & & DeviceDescr & & & & & \\
\hline & $\begin{array}{l}\text { Refractiv } \\
\text { Index }\end{array}$ & Thickness & FrameRotation & descriptior & & ModeToConsider & Incident_kpp \\
\hline 4 & 1 (air) & $0(1 / 2 \infty)$ & (none) & Line | Material \#1 & 4 & TE | TM | indifferent & $\vec{k}_{/ / \text {incident }}$ \\
\hline$\stackrel{\mathscr{E}}{=}$ & $n$ or $\left[\begin{array}{c}n e \\
\theta\end{array}\right.$ & ${ }^{e} R$ & $\begin{array}{r}\text { frame } 3 D \text { rotation } \\
\theta^{\prime} \varphi^{\prime} \gamma^{\prime} \\
\end{array}$ & Line | Material \#2 & $\stackrel{\mathscr{\Xi}}{\leftrightarrows}$ & 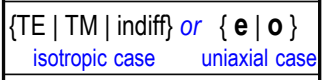 & $\begin{array}{c}=\left[k_{x, i n}, k_{y, i n}, 0\right] \\
\text { in the first }\end{array}$ \\
\hline - & $\ldots$ & $\ldots$ & ... & & $\checkmark$ & $\ldots$ & $\begin{array}{c}\text { (AbsoluteReference) } \\
\text { Frame }\end{array}$ \\
\hline$\downarrow$ & 1 (air) & $0 \quad(1 / 2 \infty)$ & ... & Line $\mid$ & $\downarrow$ & TE | TM | indifferent & \\
\hline $\begin{array}{l}L \text { differe } \\
\text { (includin } \\
\text { and exit } \\
\text { L-1 inte }\end{array}$ & $\begin{array}{l}t \text { materials } \\
\text { entrance } \\
\text { ir) } \\
\text { aces }\end{array}$ & $\begin{array}{l}\text { for uniaxial materia } \\
\text { e \& o principal indi } \\
\text { material axis direct } \\
\text { the frame used for }\end{array}$ & $\begin{array}{ll}\text { s and } & \text { along } \\
n \text { (given in } & \text { used } \\
\text { lat material) } & \end{array}$ & $\begin{array}{l}\text { in the frame } \\
\text { or that material }\end{array}$ & $\begin{array}{l}\text { the frame } r \\
\text { before ente } \\
\text { (the interfac } \\
\text { to the new }\end{array}$ & $\begin{array}{l}\text { tation takes place } \\
\text { ring the material. } \\
\text { e is perpendicular } \\
z \text { axis) }\end{array}$ & \\
\hline
\end{tabular}

\%Initializations:

CurrentFrame $\leftarrow$ AbsoluteReferenceFrame

Current_kpp $\leftarrow$ Incident_kpp \% Incident_kpp is given in the AbsoluteReferenceFrame

Ray $\leftarrow 3 D \_$Polygonal_Line with one point $([0,0,0]$ in the AbsoluteReferenceFrame $)$

Tdevice $\leftarrow 1 \%$ global "geometrical" energetic transmission

סray $\leftarrow 0 \%$ optical path along the ray

$\delta$ wave $\leftarrow 0 \%$ optical path for the wave, measured from $(0,0,0)$ in the first frame to $(0,0,0)$ in the last frame

$\%$ Computations:

for $j$ from 1 to $L-1$

- CurrentFrame $\leftrightarrow$ translate CurrentFrame from Thickness $(j)$ along (current) $z$

$\%$ Start of specific computations requested by frame rotation, which changes the $z$ direction and,

$\%$ thus, changes $\vec{k}_{/ /}$, in a different way for e and o modes in uniaxial birefringent materials.

- Compute eigenmodes $\left(\mathbf{V}_{\mathbf{0}}\right.$ matrix, $k_{z, \ell}$, etc...) for the $j$ th material and the Current_kpp in the CurrentFrame

- Compute wavevector $\vec{k}$ for the ModeToConsider $(j)$ mode in the $j$ th material in the CurrentFrame

- CurrentFrame $\leftrightarrow$ rotate CurrentFrame with FrameRotation $(j+1)$ information

- Express former wavevector $\vec{k}$ in the new CurrentFrame

- Current_kpp $\leftarrow\left(\vec{k}_{/ /}=\right.$Projection $_{x y}(\vec{k})$ in the new CurrentFrame $)$

- Compute eigenmodes $\left(\mathbf{V}_{\mathbf{A}}\right.$ matrix, $k_{z, \ell}$, etc...) for the $j$ th material and the new Current_kpp in the new CurrentFrame

- From both former eigenmode computations, keep, with appropriate transmission factors, the subjacent physical mode unchanged (same 3D $\vec{E}$ vectors) even though the mode basis have changed due to the frame rotation.

$\%$ End of specific computation due to the frame rotation

- From the (formerly computed) eigenmodes ( $\mathbf{V}_{\mathbf{A}}$ matrix, etc...) for the $j$ th material and the Current_kpp in the CurrentFrame

- Compute Poynting vector $\Re(\vec{S})$ and wavevector $\vec{k}$ for the ModeToConsider $(j)$ mode in the $j$ th material

- From $\Re(\vec{S})$ and the last point in Ray, find the intersection of the ray with the $j$ th interface, and add this point to Ray

- Dray $\leftarrow$ סray $+\left(\vec{k} / k_{0}\right) \cdot \vec{R}$ where $\vec{R}$ is a vector build from the last two points of Ray

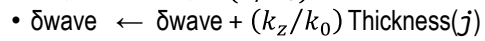

- Compute eigenmodes $\left(\mathbf{V}_{\mathbf{B}}\right.$ matrix, $k_{z, \ell}$, etc...) for the $(j+1)$ th material and the Current_kpp in the CurrentFrame

- From $\mathbf{V}_{\mathbf{A}}^{-1} \mathbf{V}_{\mathbf{B}}$ compute the generalized Fresnel transmission coefficients of the $j$ th interface, and pick the energetic transmission coefficient $T$ relevant for the ModeToConsider $(j)$ to the ModeToConsider $(j+1)$ modes. (If one or both of the ModeToConsider $(j)$ or the ModeToConsider $(j+1)$ is "indifferent", $T$ will be set to 1 )

- Tdevice $\leftarrow$ Tdevice $T$

\section{OUTPUT DATA SUMMARY :}

Ray: A polygonal line that allows to draw the ray for the ModeToConsider path.

Tdevice: The global "geometrical" energetic transmission for the ModeToConsider path סray:

The optical path along the ray for the ModeToConsider path.

סwave: The optical path for the ModeToConsider path traveled by a plane wave, measured from $(0,0,0)$ in the first frame to $(0,0,0)$ in the last frame.

Fig. 2. Synoptic flowchart of our algorithm for simulation of birefringent interferometers composed of a stack of parallel or wedged plates.

only occurs from the second plate, since both waves are ordinary in the first plate, conversely to the nominal case eo - oe. The isocontour line spacing for $20 \mu \mathrm{m}$ step is also $(\sim 1.4 \times)$ larger than in Fig. 3, meaning that fringe spacing for these wave interfer- ences is also larger. The corresponding $(o e, o o)$ crossed term will thus superimpose oblique fringes on the mainly vertical nominal ones. Note however that the moduli of the $A_{o o}$ or $A_{e e}$ complex amplitudes are much lower than the ones of $A_{\mathfrak{e o}}$ or $A_{o e}$ 


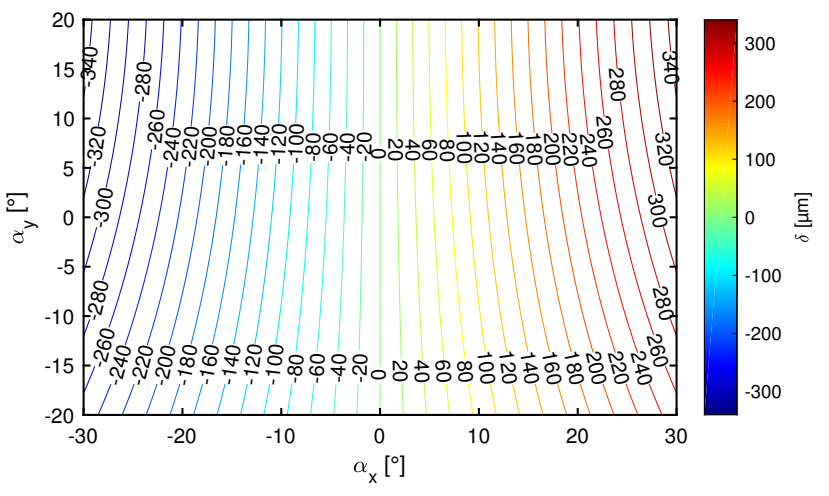

Fig. 3. Map of optical path differences (in $\mu \mathrm{m}$ ) between the nominal rays eo and oe calculated with our code, for the Savart interferometer depicted in Fig. $1\left(\lambda_{0}=0.500 \mu \mathrm{m}\right)$. This computation allows to quantify the fringe distortion of a real Savart interferometer relatively to the equispaced vertical fringes of an ideal sharing interferometer. It is worth noting that the second order approximation of the OPD as given by Eq. (12) would lead to OPD values that depart from the rigorous one by $\pm \sim 3 \mu \mathrm{m}$ in the corners of the figure.

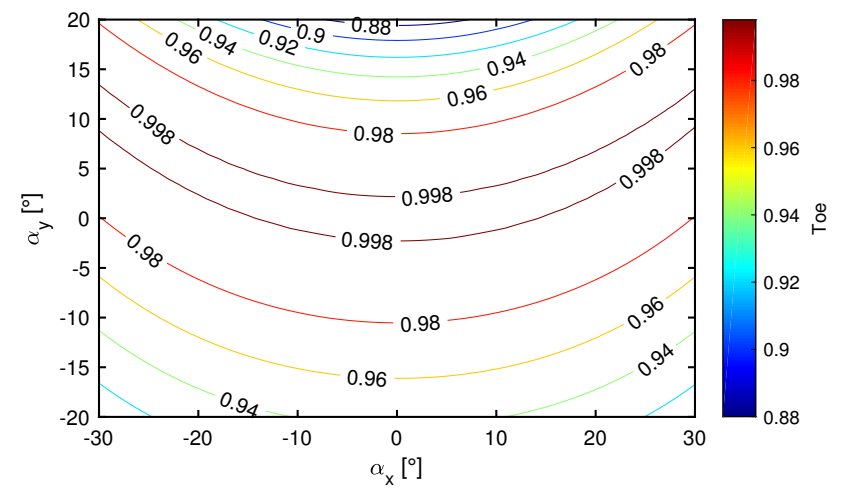

(a)

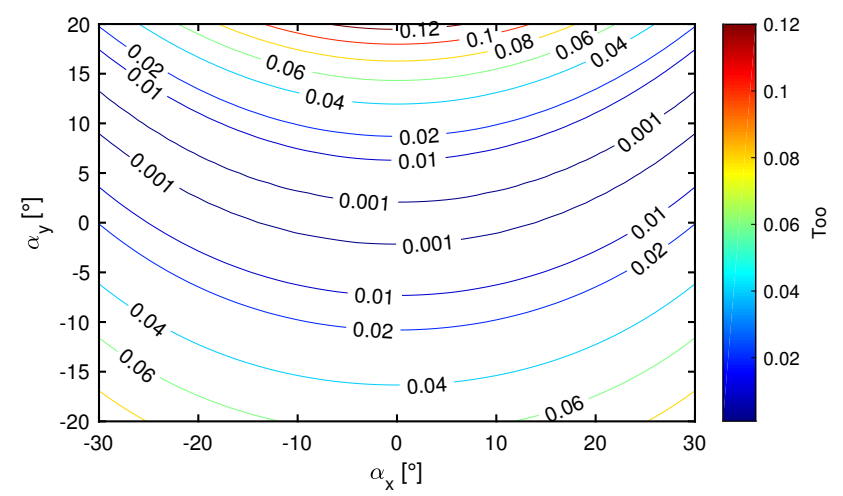

(b)

Fig. 4. Energetic transmission at the birefringent material interface versus the field angle for, (a) the nominal wave oe, and (b) the stray wave oo, for the previously described Savart interferometer example. The energetic transmission of the nominal wave $e o$ and of the stray wave ee are rather similar to the ones showed here.

as it can be inferred from $T_{o e}$ and $T_{e e}$ transmission plots on Fig. 4 . Therefore crossed terms involving one, or a fortiori two, stray

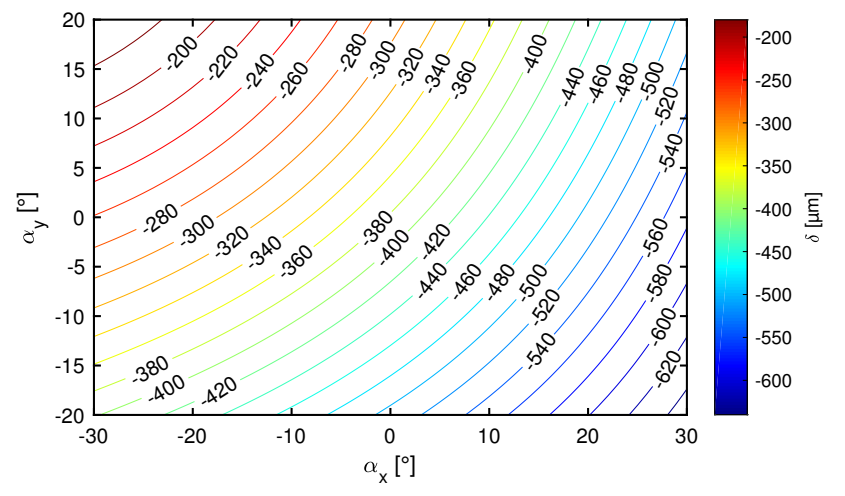

Fig. 5. Optical path difference (in $\mu \mathrm{m}$ ) between the oe and oo waves for the Savart interferometer.

waves are usually weak relatively to the crossed term between nominal waves, especially on the center part of the field of view, and the nominal fringe field is usually only sightly perturbed by such stray waves. Nevertheless, such stray wave modeling allows to quantify the fringe field perturbation, which could be useful for applications requiring large field of view and/or demanding fringe signal processing, as e.g. SFTS. In this latter case, a solution could be to extract the spectrum not by a mere Fourier transform implicitly assuming two-wave interferences, but by an inverse problem approach which takes explicitly into account the departure from a simple sinusoidal fringe field [39].

Such modeling of stray waves applies in a similar way for all birefringent interferometers consisting of birefringent material parallel plates, with all waves exiting in the same direction as the incident one. This property is no more true when using prismatic plates, like in the Double-Wollaston prism for instance, which is the subject of the following subsection.

\section{B. Case of Double-Wollaston prism device}

A Double-Wollaston (DW) interferometer [12] is presented in Fig. 6. This device is also a two-wave lateral shearing interferometer. It consists of an internal birefringent parallel plate sandwiched between two identical birefringent prisms placed head to tail; it can also be seen as a "global" parallel plate including a slanted "inner" parallel plate. As shown by 3D arrow on Fig. 6, the nominal orientation of the prism uniaxial material axis is along the prism edge, and the nominal orientation of the parallel plate uniaxial material axis is perpendicular to both the prism edge and the normal to the external interfaces ( $Z$ axis), in order that $e$ waves in the prisms essentially couple to or from $o$ waves in the inner parallel plate and vice versa. This device has two interfaces between anisotropic media, instead of just one for the Savart interferometer. Consequently, there are eight transmitted waves outgoing from the system: the two nominal waves $\{e o e, o e o\}$, the four "simply" stray waves $\{e e 0, e 00$, ooe, oee $\}$ and the two "doubly" stray waves $\{e e e, 000\}$. A key-point is that these waves do not exit the device in the same directions, conversely to the Savart device case. As long as the inner plate and the global plate are parallel plates without any residual wedges, the waves belong to three groups with respect to the exit direction (see Fig. 6): The first group of output waves consists of the two nominal waves and of the two "doubly" stray waves, which exactly keep the incident direction. The second group is made of the oee and ooe waves, and the third group is made of the eeo and eoo waves. The two specific exit directions 

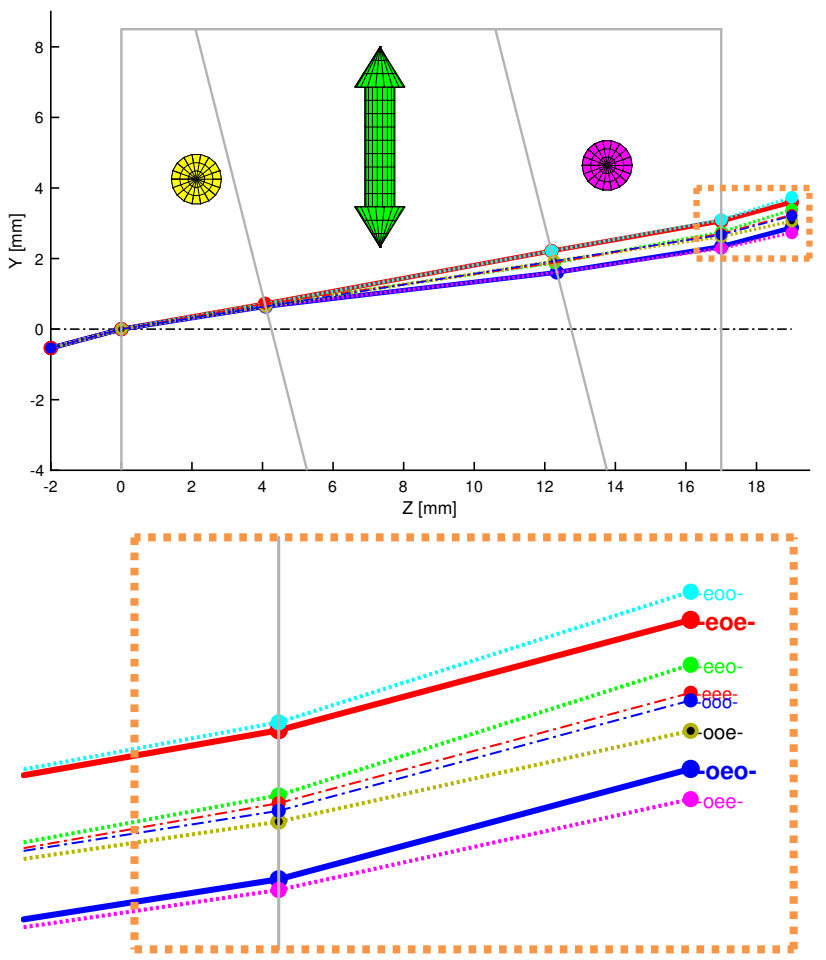

Fig. 6. Calcite Double-Wollaston interferometer, with the refraction of nominal and stray rays. The 3D arrows show the uniaxial birefringent material axis directions. The bottom plot shows a magnified view of the exiting rays with labeling. There are three different exit angles: the one of the eoe, oeo, ooo and eee rays (which is the same as the incidence angle), the one of the eeo and eoo rays and the one of the ooe and oee rays.

for the "simply" stray waves, relatively to the nominal one, lead in practice to two translated ghost images when looking at a scene at infinity through the device, a phenomenon that can be harmful for some applications even when they are weak; an experimental illustration of these ghost images will be presented in the forthcoming section.

Using our theoretical approach presented in sections 2 and 3 , we are able to compute the exit angles, the traveled optical paths and the relative intensities of the different exit waves or rays. For instance, Fig. 7a shows the evolution with the field of view of the transmission factor for the "simply" stray wave ooe ; this stray transmission is quite low, lower than for the Savart interferometer for a same field of view and a similar spectral resolution (i.e. a similar maximum OPD). Similarly, Fig. $7 \mathrm{~b}$ shows the evolution of the transmission coefficient for the "doubly" stray rays ooo ; as expected with two cascaded spurious couplings, it is much lower than the former transmission for a "simply" stray wave, and these "doubly" stray waves can usually be completely neglected for all practical purposes.

Our model is also able to compute the exit angles, OPD, and transmission coefficients when the device deviates from the ideal ones due, e.g., to manufacturing errors. As an illustration, Fig. 7c shows the transmission coefficient of the ooe wave of the former Double-Wollaston interferometer when there is a $1^{\circ}$ error (rotation about $Z$ axis) on the uniaxial material axis direction in the first birefringent prism; comparison with Fig. 7a shows a significant increase of the strength of that stray wave for some

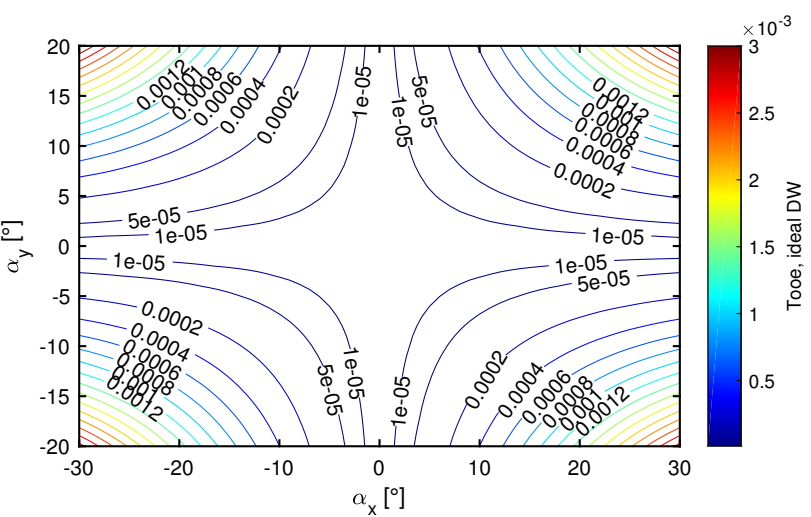

(a)

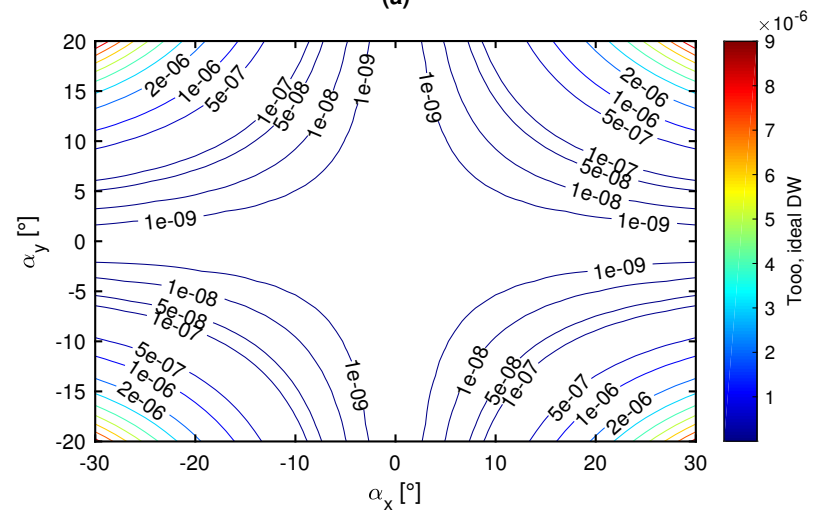

(b)

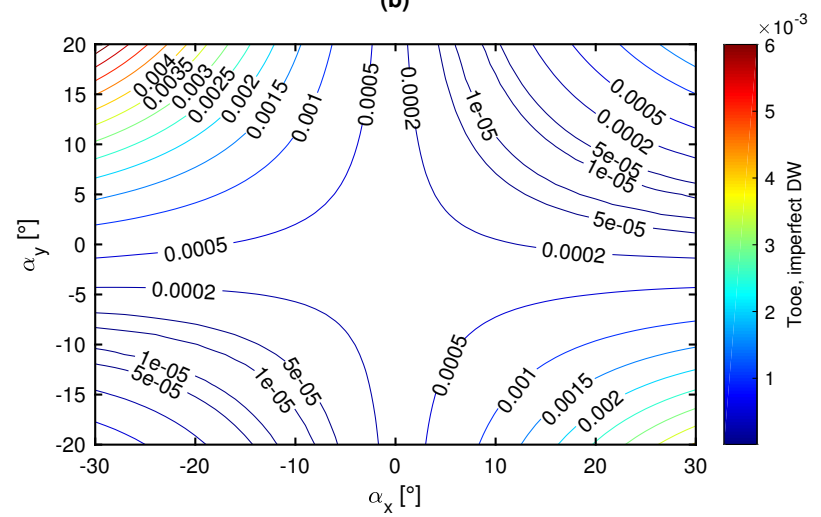

(c)

Fig. 7. Intensity of wave versus the field of view for the (nominal or imperfect) aforementioned Double-Wollaston (DW) interferometer: (a) simply stray wave ooe for nominal DW; (b) doubly stray wave ooo for nominal DW; (c) simply stray wave ooe for imperfect DW (uniaxial birefringent axis of the first material rotated by $1^{\circ}$ about $Z$ axis). 
field angles. This allows to quantitatively check the effect of such imperfections in some areas of the field of view, and, therefore, to set objective manufacturing tolerances on the interferometer components and assembly.

\section{EXPERIMENTAL STUDY OF THE DOUBLE- WOLLASTON DEVICE}
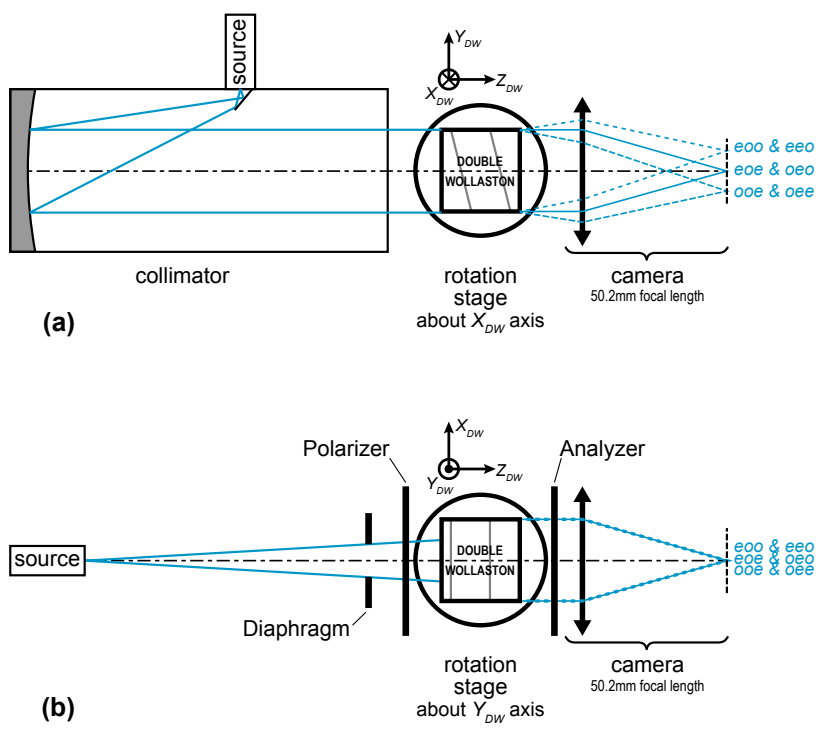

Fig. 8. Optical benches used for the experiments on the Double-Wollaston (DW) device.

(a) Setup with a collimator and no polarizer and analyzer to study the simply-stray beam directions and intensities evolution with the DW rotation about its $X$ axis.

(b) Setup with a diverging beam and polarizer and analyzer to study nominal and simply-stray fringe fields for a given DW tilt angle about its $Y$ axis.

In both cases, the camera lens is focused at infinity.

The aim here is to experimentally illustrate the presence and the properties of the stray waves in the case of a DoubleWollaston prism. For this purpose, we used a Double-Wollaston prism made of calcite, whose primary manufacturing specifications were $14.2^{\circ}$ for the prism angles, and $17 \mathrm{~mm}$ for the total thickness, as already depicted on Fig. 6 in the former section. We used a 14 bits Dalsa camera, with a focal length of $50.2 \mathrm{~mm}$ and a matrix sensor of $1024 \times 1024$ pixels with a $12 \mu \mathrm{m}$ pitch. As the sensor dynamic range did not allow to simultaneously image the nominal, the simply-stray and doubly-stray beams, we have only compared the nominal and simply-stray waves characteristics. Note also that we had only an imperfect knowledge of our Wollaston device, which furthermore had slight manufacturing errors (for instance, there was a tiny angle between the two nominal rays, which should not occur in a perfect device, and is most probably due to a lack of parallelism in the inner and/or global plates). Therefore, our goal here is to show the stray coupling phenomenon and illustrate the main behaviors of stray rays or waves in birefringent interferometers, rather than to quantitatively compare experimental measurements and theoretical predictions for tightly controlled parameters.

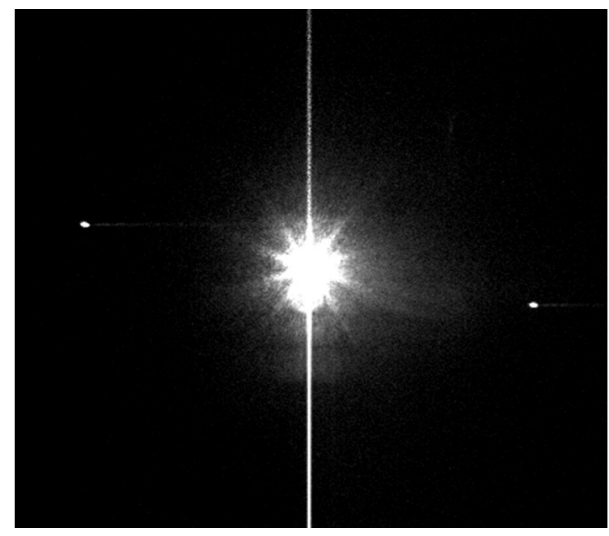

(a)

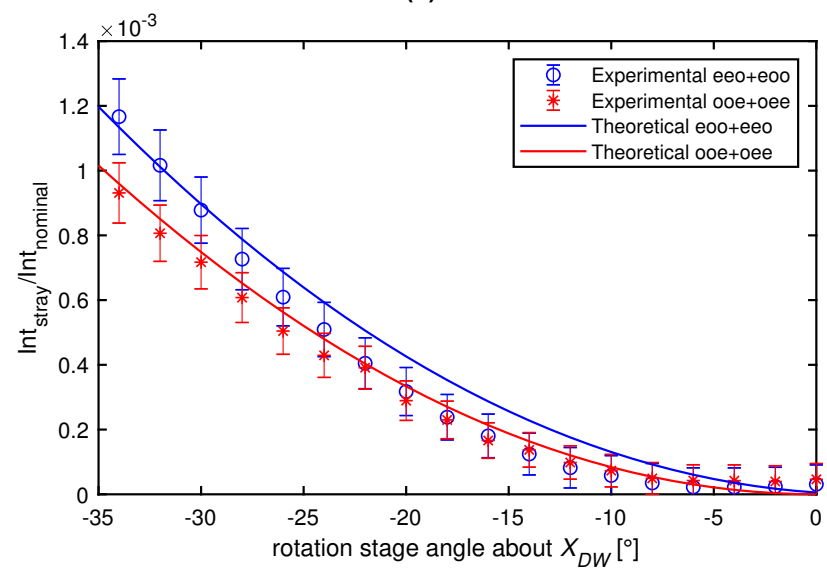

(b)

Fig. 9. (a) Camera image of the stray beams on both sides of the overexposed nominal beam, obtained in the Fig. 8a configuration for a rotation of the stage of $30^{\circ}$. (The camera had been slightly rotated about its optical axis; the "virtual" line that connects the two faint spots identifies the DW $Y$ direction).

(b) Experimental and theoretical ratios of the stray and nominal spot intensities versus the rotation stage angle (about DW $X$ axis) for the same Fig. 8a configuration. The theoretical curves have been obtained for an adjusted field angle $\alpha_{x}$ of $8.3^{\circ}$ introduced in the collimator orientation. (The rotation stage angle is somewhat equivalent to an $\alpha_{y}$ field angle).

\section{A. Characterization of simply stray waves in intensity}

The first optical bench used is presented on Fig. 8a. It is made of an illumination source (laser or LED) placed in front of a collimator, followed by the Double-Wollaston device mounted on rotatory stage and the Dalsa camera focused at infinity. When illuminated by a point source, a very bright spot appears on the camera, flanked by two faint replica, as it can be seen on Fig. 9a.

The experimental centroid position of the $\{$ ooe, oee $\}$ spot in the camera sensor plane for Fig. 9a is $2.07 \mathrm{~mm}$ apart from the centroid of the nominal spots whereas the theoretical distance, as evaluated with the camera lens focal length and the computed exit angle, is $2.08 \mathrm{~mm}$, which leads to a relative error of approximately $0.5 \%$. We have the same amount of relative error for the $\{e 00, e e 0\}$ spot.

The intensity of the stray waves have been measured for different positions of the rotatory stage. The experimental ratios of the stray spot intensities to the nominal spot intensity are 


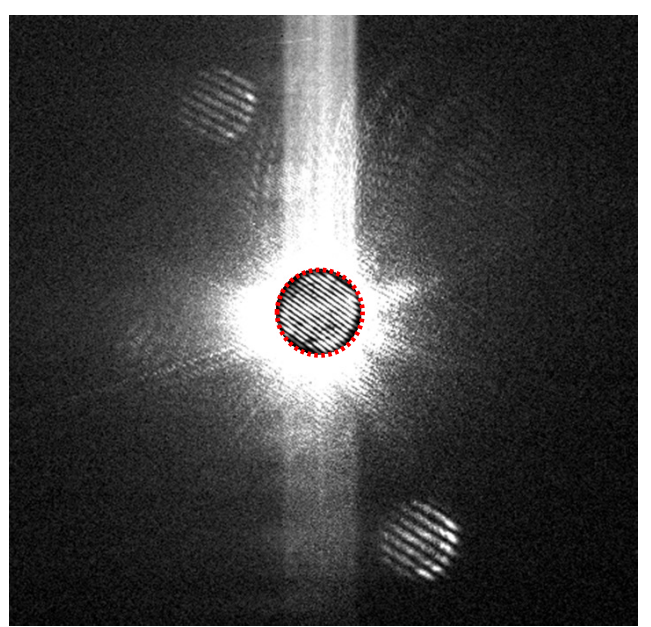

(a)

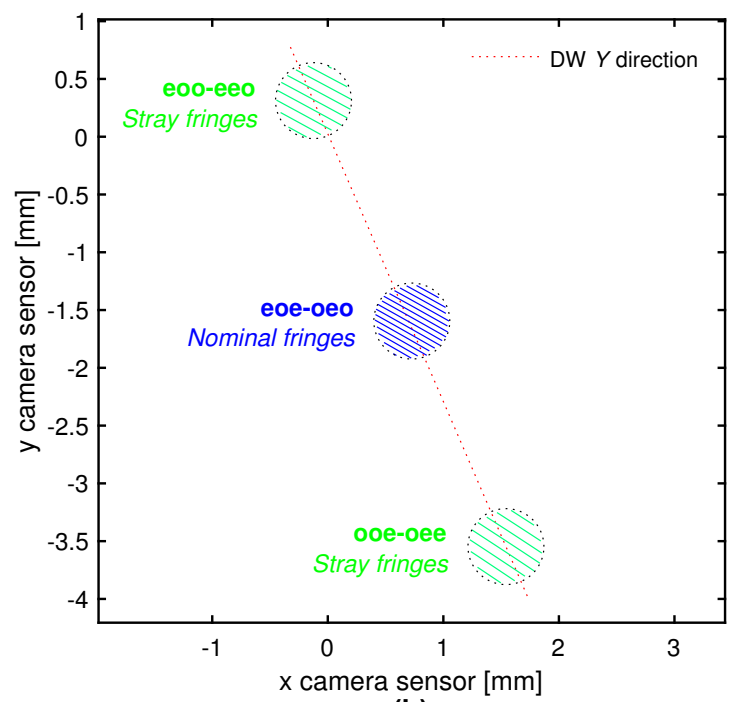

(b)

Fig. 10. (a) Experimental image of the stray and nominal fringes obtained in the Fig. $8 \mathrm{~b}$ configuration, with a diverging beam limited to $\varnothing 0.75^{\circ}$ by a suitable diaphragm, for a rotation stage angle of $-30^{\circ}$. The central area is for illustrative purpose only: it is actually an inset from another image, taken in quite similar conditions, but with a much lower power of the source to avoid overexposure. Fringes have been obtained with a $594 \mathrm{~nm}$ laser of sufficient coherence length. Indeed, the order of interference of the nominal fringes is around 250 (a somewhat high value resulting from the large $-30^{\circ}$ rotation angle), whereas the order of the stray fringes is around 2600. The camera has been rotated by $\sim-25^{\circ}$ about its optical axis in order that the blooming due to the central overexposure does not alter the images of the stray fringes.

(b) Fringe simulation (isocontours of OPD $/ \lambda_{0}$ with step 1 ) as given by our program for a rotation stage angle of $-30.0^{\circ}$ and an adjusted $\alpha_{y}$ value of $-2.0^{\circ}$. (Precise comparison of the central area fringe shape with the one in the inner inset above is pointless due to slight modifications of the observation conditions between the acquisitions of the two pictures).

presented on Fig. 9b. We have also represented the theoretical values. The general trends of the experimental measurements and the theoretical computations are in good agreement. The remaining discrepancies between theoretical and experimental values may reasonably be attributed to measurements uncertainties and also imperfect DW device parameter knowledge. In particular, introducing a (somewhat hypothetical) small error in the uniaxial material axis orientation of the DW inner plate, as for Fig. 7c, may explain non-zero values of the intensity ratios for the $0^{\circ}$ rotation stage angle and allows better general fitting of the theoretical curves to the experimental data for an adjusted value.

\section{B. Characterization of stray fringes}

For this experiment, Fig. $8 \mathrm{~b}$ configuration is used. The optical bench is composed of a point source and a diaphragm to limit the divergence of the beam. Then follow the polarizer, the Double-Wollaston plate, the analyzer and the camera, still focused at infinity. The limited beam angle allows to observe separately the different families of fringes. As Fig. 10a shows, the stray fringes between $\{e e 0, e 00\}$ rays and $\{$ ooe, oee $\}$ can be experimentally seen for a sufficient power of the source, just like the nominal fringes for a much lower power. These fringes, for a $-30^{\circ}$ rotation of the DW device about its $Y$ axis, are no more perpendicular to the DW $Y$ direction as for small field angles (the fringe shape of a DW device is complex at large field of view). Fig. 10b shows a theoretical computation of the fringe field for the same configuration as the Fig. 10a experimental one. This shows that our model is able to effectively predict the stray waves interference for such a Double-Wollaston interferometer.

\section{CONCLUSION}

Based on Maxwell's equations, we have developed a theoretical framework and a computer program to simulate the propagation of plane waves in an homogeneous anisotropic medium and at the interface between two anisotropic media. This tool can be used to model various birefringent devices, like, for instance, Savart plates or double-Wollaston prism interferometers, but also much more complex interferometers, provided that all surfaces are plane. For our application on hyperspectral imaging based on SFTS, this tool is particularly helpful to compute the exact OPD for birefringent interferometers, whatever be the field angles or the complexity of the interferometer, including misorientation of the surfaces or of the optical axes of the birefringent materials. In contrast, the analytical expression of the OPD introduced by a parallel plate given by references [34, 35], is only usable for assemblies of perfect parallel plates, and few, if any, papers have presently, to our knowledge, addressed the general case of an arbitrary stack of wedged birefringent plates without approximation in the OPD computation. Therefore our tool proves to be useful to analyze large field of view behavior and to evaluate, or possibly to minimize, the impacts of manufacturing errors. Our electromagnetic approach also has the advantage to allow the evaluation of the intensity of all the transmitted rays, whether they are expected or spurious. Indeed, two-wave birefringent interferometers usually rely on an assembly of several birefringent plates. When the coupling between the desired ordinary or extraordinary modes is perfect, only two rays leave the interferometer; however, this perfect coupling only occurs for specific incidence angles and perfect orientations of the material uniaxial optical axes, and in the general case, stray rays will disturb the measured interferogram, by creating either stray fringes, or ghost images if prisms are present. These stray rays are all the more significant since the field angle is wide, as we 
have experimentally illustrated. But for lateral shearing interferometers, wide field of view is a way to reduce the thickness of the interferometer [11]. It is therefore important to anticipate the impact of these stray rays, both in terms of intensity and of OPD, in order to properly decide on dimensions and tolerances of the device components and their assembly at the design stage.

\section{ACKNOWLEDGMENTS}

The authors thank Jean-Paul Hugonin from the Nanophotonics and Electromagnetism team of the Laboratoire Charles Fabry for useful discussions.

\section{REFERENCES}

1. D. Cabib, R. Buckwald, M. Lavi, and A. Gil, "New airborne pushbroom spectral imager for the 3-5 and 7-12 $\mu \mathrm{m}$ wavelength ranges," in "Conference Proceedings of the XXXI Symposium of the Optical Society of India, ICOL-2005, Dehradun, India," (2005).

2. S. Crites, P. Lucey, R. Wright, H. Garbeil, K. Horton, and M. Wood, "A low cost thermal infrared hyperspectral imager for small satellites," in "SPIE Defense, Security, and Sensing, Proc. SPIE 8385," (2012), pp. 838509-838509.

3. A. Barducci, F. Castagnoli, G. Castellini, D. Guzzi, C. Lastri, P. Marcoionni, V. Nardino, and I. Pippi, "Developing a new hyperspectral imaging interferometer for Earth observation," Optical Engineering 51, 111706 (2012).

4. L. Su, Y. Yuan, B. Xiangli, F. Huang, J. Cao, L. Li, and S. Zhou, "Spectrum reconstruction method for airborne temporally spatially modulated Fourier transform imaging spectrometers," IEEE Transactions on Geoscience and Remote Sensing 52, 3720-3728 (2014).

5. Y. Ferrec, J. Taboury, H. Sauer, P. Chavel, P. Fournet, C. Coudrain, J. Deschamps, and J. Primot, "Experimental results from an airborne static Fourier transform imaging spectrometer," Applied Optics 50, 5894-5904 (2011).

6. C. Coudrain, S. Bernhardt, M. Caes, R. Domel, Y. Ferrec, R. Gouyon, D. Henry, M. Jacquart, A. Kattnig, P. Perrault et al., "SIELETERS, an airborne infrared dual-band spectro-imaging system for measurement of scene spectral signatures," Optics express 23, 16164-16176 (2015).

7. Y. Ferrec, N. Ayari-Matallah, P. Chavel, F. Goudail, H. Sauer, J. Taboury, J.-C. Fontanella, C. Coudrain, and J. Primot, "Noise sources in imaging static Fourier transform spectrometers," Optical Engineering 51, 111716 (2012).

8. T. Svensson, D. Bergström, L. Axelsson, M. Fridlund, and T. Hallberg, "Design, calibration and characterization of a low-cost spatial Fourier transform LWIR hyperspectral camera with spatial and temporal scanning modes," in "Proc.SPIE," , vol. 10644 (2018), vol. 10644, pp. 1 15.

9. G. Zhan, K. Oka, T. Ishigaki, and N. Baba, "Birefringent imaging spectrometer," Applied Optics 41, 734-738 (2002).

10. C. Zhang, B. Xiangli, B. Zhao, and X. Yuan, "A static polarization imaging spectrometer based on a Savart polariscope," Optics Communications 203, 21-26 (2002).

11. A. Pola Fossi, Y. Ferrec, N. Roux, O. D'Almeida, N. Guerineau, and $\mathrm{H}$. Sauer, "Miniature and cooled hyperspectral camera for outdoor surveillance applications in the mid-infrared," Optics letters (2016).

12. S. Prunet, B. Journet, and G. Fortunato, "Exact calculation of the optical path difference and description of a new birefringent interferometer," Optical Engineering 38, 983-990 (1999).

13. J. Li, J. Zhu, C. Qi, C. Zheng, B. Gao, Y. Zhang, and X. Hou, "Compact static imaging spectrometer combining spectral zooming capability with a birefringent interferometer," Optics express 21, 10182-10187 (2013).

14. T. Okamoto, S. Kawata, and S. Minami, "A photodiode array Fourier transform spectrometer based on a birefringent interferometer," Applied spectroscopy 40, 691-695 (1986).

15. M. Padgett and A. Harvey, "A static Fourier-transform spectrometer based on Wollaston prisms," Review of scientific instruments 66, 28072811 (1995).
16. W. H. Smith and P. D. Hammer, "Digital array scanned interferometer: sensors and results," Applied optics 35, 2902-2909 (1996).

17. M. W. Kudenov and E. L. Dereniak, "Compact real-time birefringent imaging spectrometer," Optics express 20, 17973-17986 (2012).

18. B. D. Maione, D. Luo, M. Miskiewicz, M. Escuti, and M. W. Kudenov, "Spatially heterodyned snapshot imaging spectrometer," Appl. Opt. 55, 8667-8675 (2016).

19. A. Oriana, J. Réhault, F. Preda, D. Polli, and G. Cerullo, "Scanning Fourier transform spectrometer in the visible range based on birefringent wedges," J. Opt. Soc. Am. A 33, 1415-1420 (2016).

20. A. Hegyi, "Birefringent static Fourier-transform spectrometer for flow cytometry and imaging spectroscopy," Opt. Express 25, 17402-17410 (2017).

21. J. Craven-Jones, M. Kudenov, M. Stapelbroek, and E. Dereniak, "Infrared hyperspectral imaging polarimeter using birefringent prisms," Applied Optics 50, 1170-1185 (2011).

22. T.-H. Tsai and D. Brady, "Coded aperture snapshot spectral polarization imaging," Applied optics 52, 2153-2161 (2013).

23. M. Born and E. Wolf, Principles of optics: electromagnetic theory of propagation, interference and diffraction of light, Chapter 15, Section 15.1 (Cambridge university press, 1999).

24. S. Teitler and B. Henvis, "Refraction in stratified, anisotropic media," JOSA 60, 830-834 (1970).

25. D. W. Berreman, "Optics in stratified and anisotropic media: $4 \times 4$ matrix formulation," JOSA 62, 502-510 (1972).

26. I. Abdulhalim, "Analytic propagation matrix method for linear optics of arbitrary biaxial layered media," Journal of Optics A: Pure and Applied Optics 1, 646 (1999).

27. P. Yeh, "Electromagnetic propagation in birefringent layered media," JOSA 69, 742-756 (1979).

28. P. Yeh, "Optics of anisotropic layered media: a new $4 \times 4$ matrix algebra," Surface Science 96, 41-53 (1980).

29. H. Wöhler, G. Haas, M. Fritsch, and D. Mlynski, "Faster $4 \times 4$ matrix method for uniaxial inhomogeneous media," JOSA A 5, 1554-1557 (1988).

30. J. D. Jackson, Classical electrodynamics, 3rd edition (Wiley New York, 1999).

31. M. Born and E. Wolf, Principles of optics: electromagnetic theory of propagation, interference and diffraction of light, Chapter 1, Section 1.1.3 (Cambridge university press, 1999).

32. M. T. Garea and M. C. Simon, "Interference patterns in interferometers of birefringent uniaxial wedges," in "Proc.SPIE 2730," (1996), pp. 220223.

33. J. Li, H. Wu, and C. Qi, "Complete description of the optical path difference of a novel spectral zooming imaging spectrometer," Optics Communications 410, 598 - 603 (2018).

34. M. Françon and S. Mallick, Polarization interferometers (Wiley Interscience, 1971). Appendix B.

35. F. E. Veiras, L. I. Perez, and M. T. Garea, "Phase shift formulas in uniaxial media: an application to waveplates," Appl. Opt. 49, 27692777 (2010).

36. K. Oka and N. Saito, "Snapshot complete imaging polarimeter using Savart plates," in "SPIE Optics+ Photonics," (International Society for Optics and Photonics, 2006), pp. 629508-629508.

37. H. Luo, K. Oka, E. DeHoog, M. Kudenov, J. Schiewgerling, and E. L. Dereniak, "Compact and miniature snapshot imaging polarimeter," Applied optics 47, 4413-4417 (2008).

38. N. Murakami and N. Baba, "Common-path lateral-shearing nulling interferometry with a Savart plate for exoplanet detection," Optics letters 35, 3003-3005 (2010).

39. F. Gillard, S. Lefebvre, Y. Ferrec, L. Mugnier, S. Rommeluère, C. Benoït, N. Guérineau, and J. Taboury, "Inverse problem approaches for stationary Fourier transform spectrometers," Optics letters 36, 2444-2446 (2011). 